\title{
Nowhere-zero $k$-flows of supergraphs
}

\author{
Bojan Mohar* and Riste Š krekovski* \\ Department of Mathematics, \\ University of Ljubljana, \\ Jadranska 19, 1111 Ljubljana \\ Slovenia \\ bojan.mohar@uni-lj.si \\ riste.skrekovski@fmf.uni-lj.si
}

Submitted: March 28, 2000; Accepted: January 30, 2001.

Mathematical Subject Classification: 05C15, 05C99.

\begin{abstract}
Let $G$ be a 2-edge-connected graph with $o$ vertices of odd degree. It is well-known that one should (and can) add $\frac{o}{2}$ edges to $G$ in order to obtain a graph which admits a nowhere-zero 2-flow. We prove that one can add to $G$ a set of $\leq\left\lfloor\frac{o}{4}\right\rfloor,\left\lceil\frac{1}{2}\left\lfloor\frac{o}{5}\right\rfloor\right\rceil$, and $\left\lceil\frac{1}{2}\left\lfloor\frac{o}{7}\right\rfloor\right\rceil$ edges such that the resulting graph admits a nowhere-zero 3-flow, 4-flow, and 5-flow, respectively.
\end{abstract}

\section{Introduction}

Graphs in this paper may contain multiple edges and loops. A vertex of $G$ is odd (even) if its degree is odd (even). We denote by $o(G)$ the number of odd vertices of $G$. Let $G$ be a graph such that no component of $G$ is a cycle. Then there is a unique graph $G^{\prime}$ which is homeomorphic to $G$ and has no vertices of degree 2. We say that $G^{\prime}$ is obtained from $G$ by suppressing vertices of degree 2 , and we denote this by $G^{\prime} \propto G$.

Let $\Gamma$ be an Abelian group, let $D$ be an orientation of a graph $G$ and $f: E(G) \rightarrow \Gamma$. The pair $(D, f)$ is a $\Gamma$-flow in $G$ if the following condition is satisfied at every vertex $v \in V(G)$ :

$$
\sum_{e \in E^{+}(v)} f(e)=\sum_{e \in E^{-}(v)} f(e)
$$

\footnotetext{
*Supported in part by the Ministry of Science and Technology of Slovenia, Research Project J1-05020101-99.
} 
where $E^{+}(v)$ and $E^{-}(v)$ denote the sets of outgoing and ingoing edges (with respect to the orientation $D$ ) incident with $v$, respectively.

A flow $(D, f)$ is nowhere-zero if $f(e) \neq 0$ for every $e \in E(G)$. If $\Gamma \cong \mathbb{Z}$ and $-k<$ $f(e)<k$ then $(D, f)$ is a $k$-flow. The concept of nowhere-zero flows was introduced and studied by Tutte [9]. For a 2-edge-connected graph $G$ and a group $\Gamma$ of order $k$, Tutte [8] proved that $G$ admits a nowhere-zero $k$-flow if and only if it admits a nowhere-zero $\Gamma$-flow. Seymour [7] proved that every 2-edge-connected graph admits a nowhere-zero 6-flow. We refer to [10] for further results on flows in graphs.

Let $\phi_{k}^{+}(G)$ be the minimum number of edges whose addition to $G$ gives rise to a graph which admits a nowhere-zero $k$-flow. Similarly, let $\phi_{k}^{-}(G)$ be the minimum number of edges whose deletion from $G$ leaves a graph with a nowhere-zero $k$-flow. Clearly, $\phi_{k}^{+}(G) \leq \phi_{k}^{-}(G)$ since we achieve a similar effect by doubling an edge as we do by deleting it.

Let $G$ be a 2-edge-connected graph with $o=o(G)$ vertices of odd degree. It is obvious that we should and that we can add $\frac{o}{2}$ edges to $G$ in order to obtain an Eulerian graph, i.e. a graph which admits a nowhere-zero 2-flow. Thus, $\phi_{2}^{+}(G)=\frac{o}{2}$. We shall prove that $\phi_{3}^{+}(G) \leq\left\lfloor\frac{o}{4}\right\rfloor, \phi_{4}^{+}(G) \leq\left\lceil\frac{1}{2}\left\lfloor\frac{o}{5}\right\rfloor\right\rceil$, and $\phi_{5}^{+}(G) \leq\left\lceil\frac{1}{2}\left\lfloor\frac{o}{7}\right\rfloor\right\rceil$, respectively. It is also shown that upper bounds which are linear in $o(G)$ are best possible for 3-flows and 4-flows. They are also best possible for 5 -flows if the Tutte 5-Flow-Conjecture is not true (otherwise $\phi_{5}(G)=0$ for 2-edge-connected graphs). Some additional comments on the tightness and importance of these bounds are collected at the end of the paper.

We will use the following lemma of Fleischner [4] (see also [10]):

Lemma 1.1 (Splitting Lemma) Let $G$ be a 2-edge-connected graph, let $v$ be a vertex of $G$ of degree $\geq 4$, and let $e_{0}, e_{1}, e_{2}$ be edges incident with $v$ which do not form an edge-cut of $G$. Let $G_{i}(i=1,2)$ be the graph constructed from $G$ by splitting $v$ into vertices $v_{1}$ and $v_{2}$ such that $v_{1}$ is incident with $e_{0}$ and $e_{i}$ and $v_{2}$ is incident with all other edges at $v$. Then one of $G_{1}$ and $G_{2}$ is 2-edge-connected.

\section{3 -flows}

Theorem 2.1 Let $G$ be a loopless cubic multigraph on $n$ vertices. Then, $\phi_{3}^{+}(G) \leq\left\lfloor\frac{n}{4}\right\rfloor$.

Proof. Suppose that the theorem is false and $G$ is a counterexample with minimum number of vertices. Suppose that $G=G_{1} \cup G_{2}$, where $G_{1}$ and $G_{2}$ are vertex disjoint graphs. Let $n_{i}=\left|V\left(G_{i}\right)\right|, i=1,2$. By the minimality

$$
\phi_{3}^{+}(G) \leq \phi_{3}^{+}\left(G_{1}\right)+\phi_{3}^{+}\left(G_{2}\right) \leq\left\lfloor\frac{n_{1}}{4}\right\rfloor+\left\lfloor\frac{n_{2}}{4}\right\rfloor \leq\left\lfloor\frac{n_{1}+n_{2}}{4}\right\rfloor .
$$

This shows that $G$ is connected. Let $C=v_{1} v_{2} \cdots v_{k} v_{1}$ be a shortest cycle in $G$. For $i=1, \ldots, k$, denote by $v_{i}^{\prime}$ the neighbor of $v_{i}$ distinct from $v_{i+1}$ and $v_{i-1}$. (All indices are considered modulo $k$.) If $v_{i}^{\prime}$ does not exist, then $k=2$ and $G$ has two vertices only. In this case, the claim clearly holds. 
If $k=2$, choose $C$ such that $v_{1}^{\prime} \neq v_{2}^{\prime}$ whenever possible. If $v_{1}^{\prime} \neq v_{2}^{\prime}$, let $G^{\prime}=$ $G-V(C)+v_{1}^{\prime} v_{2}^{\prime}$. By the induction hypothesis, there is a set $F$ of at most $\left\lfloor\frac{n-2}{4}\right\rfloor$ edges such that $G_{F}^{\prime}:=G^{\prime}+F$ has a nowhere-zero 3-flow. Then, clearly $G_{F}:=G+F$ also has a nowhere-zero 3 -flow.

In the sequel we shall apply the induction hypothesis several times. We shall always denote by $G^{\prime}$ the smaller graph and then use $F, G_{F}^{\prime}$, and $G_{F}$ in the same way as above.

Suppose now that $k=2$ and $v_{1}^{\prime}=v_{2}^{\prime}$. Let $v_{1}^{\prime \prime}$ be the neighbor of $v_{1}^{\prime}$ distinct from $v_{1}$ and $v_{2}$. Let $G^{\prime}$ be the cubic graph which is homeomorphic to $G-\left\{v_{1}, v_{2}, v_{1}^{\prime}\right\}$. Then $G^{\prime}$ has $n-4$ vertices. If it had a loop, then the two edges of $G-\left\{v_{1}, v_{2}, v_{1}^{\prime}\right\}$ incident with $v_{1}^{\prime \prime}$ would be parallel edges, and we would choose them as the cycle $C$ since their neighbors are distinct vertices. Therefore, $G^{\prime}$ is loopless, and we can apply the induction hypothesis to $G^{\prime}$. It is easy to see that a nowhere-zero 3 -flow of $G_{F}^{\prime}$ can be extended to a nowhere-zero 3-flow of $G_{F}+v_{1}^{\prime \prime} v_{1}$.

Suppose now that $k=3$. If $v_{1}^{\prime}=v_{2}^{\prime}=v_{3}^{\prime}$, then $G=K_{4}$ for which $\phi_{3}^{+}\left(K_{4}\right)=1$. Assume now that $v_{1}^{\prime}=v_{2}^{\prime} \neq v_{3}^{\prime}$. Let $v_{1}^{\prime \prime}$ be as above. If $v_{1}^{\prime \prime} \neq v_{3}^{\prime}$, let $G^{\prime} \propto G-V(C)-v_{1}^{\prime}+v_{3}^{\prime} v_{1}^{\prime \prime}$. We apply the induction hypothesis to $G^{\prime}$ and get an edge set $F,|F| \leq\left\lfloor\frac{n-4}{4}\right\rfloor$, such that $G_{F}^{\prime}$ has a nowhere-zero 3-flow. Finally, the nowhere-zero 3-flow of $G_{F}^{\prime}$ can be extended to a nowhere-zero 3 -flow of $G_{F}+v_{1} v_{3}^{\prime}$. If $v_{1}^{\prime \prime}=v_{3}^{\prime}$, let $G^{\prime} \propto G-V(C)-v_{1}^{\prime}-v_{1}^{\prime \prime}$. Let $v_{1}^{\prime \prime \prime}$ be the third neighbor of $v_{1}^{\prime \prime}$. It is easy to see that the flow of $G_{F}^{\prime}$ can be extended to a nowhere-zero 3-flow of $G_{F}+v_{1}^{\prime \prime \prime} v_{1}$.

The remaining case for $k=3$ is when $v_{1}^{\prime}, v_{2}^{\prime}, v_{3}^{\prime}$ are all distinct. Here we let $G^{\prime} \propto$ $G-V(C)+v_{2}^{\prime} v_{3}^{\prime}$. Again, $G^{\prime}$ is a loopless cubic graph on $n-4$ vertices and the 3-flow of $G_{F}^{\prime}$ can be extended to $G_{F}+v_{1}^{\prime} v_{2}$.

From now on we assume that $k \geq 4$. First we deal with the case when $v_{i}^{\prime}=v_{j}^{\prime}$, for a pair of distinct indices $i, j, 1 \leq i<j \leq k$. We may assume that $i=1$ and $j \leq\left\lceil\frac{k+1}{2}\right\rceil$. Consider the cycle $C^{\prime}=v_{1} v_{2} \cdots v_{j} v_{1}^{\prime} v_{1}$. Since its length is $j+1 \geq k$, we get $k=4$ and $j=3$. We have two subcases. First, suppose that also $v_{2}^{\prime}=v_{4}^{\prime}$. Let $G^{\prime} \propto G-V(C)-v_{1}^{\prime}-v_{2}^{\prime}$ (if $v_{1}^{\prime \prime} \neq v_{2}^{\prime \prime}$ ), and $G^{\prime} \propto G-V(C)-v_{1}^{\prime}-v_{2}^{\prime}-v_{1}^{\prime \prime}$ (if $v_{1}^{\prime \prime}=v_{2}^{\prime \prime}$ ), respectively. If $v_{1}^{\prime \prime}=v_{2}^{\prime}$, then also $v_{2}^{\prime \prime}=v_{1}^{\prime}$ and $G=K_{3,3}$. Since $K_{3,3}$ has a nowhere-zero 3-flow, we may assume that $v_{1}^{\prime \prime} \neq v_{2}^{\prime}$ and that $G^{\prime}$ is nonempty. It has $n-8$ vertices. It is easy to see that $G^{\prime}$ has no loops (otherwise it would have a cycle of length $\leq 3$ ). Now, $G_{F}+v_{1}^{\prime \prime} v_{2}^{\prime}+v_{1}^{\prime} v_{2}^{\prime \prime}$ and $G_{F}+v_{1}^{\prime \prime \prime} v_{1}^{\prime}+v_{1}^{\prime \prime} v_{1}^{\prime}$ (respectively) admits an extension of the flow of $G_{F}^{\prime}$ to a nowhere-zero 3-flow.

The second subcase is when $v_{1}^{\prime}=v_{3}^{\prime}$ but $v_{2}^{\prime} \neq v_{4}^{\prime}$. We may assume that $v_{1}^{\prime \prime} \neq v_{2}^{\prime}$. Let $G^{\prime} \propto G-V(C)-v_{1}^{\prime}+v_{1}^{\prime \prime} v_{2}^{\prime}$. By the induction hypothesis, there is an edge set $F$, $|F| \leq\left\lfloor\frac{n-6}{4}\right\rfloor$, such that $G_{F}^{\prime}$ has a nowhere-zero 3-flow. This flow can be extended to a nowhere-zero 3 -flow in $G_{F}+v_{2} v_{4}^{\prime}$.

From now on we may assume that $v_{i}^{\prime} \neq v_{j}^{\prime}$ if $i \neq j$. If $k$ is even, put $G^{\prime} \propto G-V(C)$. If $k$ is odd, let $G^{\prime} \propto G-V(C)+v_{1}^{\prime} v_{2}^{\prime}$. Suppose that $G^{\prime}$ has a loop. A loop in $G^{\prime}$ corresponds to a cycle $C^{\prime}$ of $G$ such that precisely one vertex of $C^{\prime}$ has degree 3 in $G-V(C)$ (or $\left.G-V(C)+v_{1}^{\prime} v_{2}^{\prime}\right)$, and other vertices of $C^{\prime}$ have degree 2. Since $C^{\prime}$ has length $\geq k$, it contains a path $P^{\prime}$ of length $k-2$ such that $V\left(P^{\prime}\right) \subseteq\left\{v_{1}^{\prime}, \ldots, v_{k}^{\prime}\right\}$.

Suppose that $v_{i}^{\prime} v_{j}^{\prime} \in E\left(P^{\prime}\right)$. Then $v_{i} v_{i}^{\prime} v_{j}^{\prime} v_{j}$ and a segment of $C$ form a cycle in $G$ of 
length $\leq \frac{k}{2}+3$. This implies that $\frac{k}{2}+3 \geq k$, i.e. $k \leq 6$. If $k=6$, then $i=j \pm 3$, so $P^{\prime}$ cannot exist. Similarly, if $k=5$, then $P^{\prime}=v_{i}^{\prime} v_{i+2}^{\prime} v_{i+4}^{\prime} v_{i+6}^{\prime}$ (indices modulo 5). In particular, $V\left(P^{\prime}\right)$ contains either $v_{1}^{\prime}$ or $v_{2}^{\prime}$. A contradiction, since $v_{1}^{\prime}$ and $v_{2}^{\prime}$ have degree 3 in $G-V(C)+v_{1}^{\prime} v_{2}^{\prime}$. The remaining possibility is when $k=4$. In that case, we let $G^{\prime \prime} \propto G-V(C)+v_{1}^{\prime} v_{2}^{\prime}+v_{3}^{\prime} v_{4}^{\prime}$ and apply the induction hypothesis on $G^{\prime \prime}$. The resulting nowhere-zero 3-flow in $G_{F}^{\prime \prime}$ either gives rise to a nowhere-zero 3-flow in $G_{F}$ or in $G_{F}+v_{1} v_{3}$.

Now, we return to the general case where we may assume that $G^{\prime}$ is loopless. Observe that $n-\left|V\left(G^{\prime}\right)\right|=4\left\lfloor\frac{k}{2}\right\rfloor$. So, after applying the induction hypothesis to $G^{\prime}$, we may add further $\left\lfloor\frac{k}{2}\right\rfloor$ edges to $G_{F}$ in order to get a graph with a nowhere-zero 3-flow. If $k$ is even, we add the edges $v_{1}^{\prime} v_{2}^{\prime}, v_{3}^{\prime} v_{4}^{\prime}, \ldots, v_{k-1}^{\prime} v_{k}^{\prime}$. If $k$ is odd we add the edges $v_{3}^{\prime} v_{4}^{\prime}, \ldots, v_{k-2}^{\prime} v_{k-1}^{\prime}$, and $v_{k}^{\prime} v_{1}$. In both cases, it is easy to see that a nowhere-zero 3-flow of $G_{F}^{\prime}$ gives rise to a nowhere-zero 3 -flow in $G_{F}$ with the additional $\left\lfloor\frac{k}{2}\right\rfloor$ edges.

By Lemma 1.1 and Theorem 2.1 we obtain the following result.

Corollary 2.2 Let $G$ be a 2-edge-connected multigraph with $o=o(G)$ odd vertices. Then we can add $\leq\left\lfloor\frac{o}{4}\right\rfloor$ edges such that the new graph $G$ admits a nowhere-zero 3-flow.

\section{4-flows}

The next lemma known as Parity Lemma is due to Blanuša [2].

Lemma 3.1 (Parity Lemma) Let $G$ be a cubic graph and let $c: E(G) \rightarrow\{1,2,3\}$ be an edge-coloring of $G$. If a cutset $T$ consists of $n$ edges such that $n_{i}$ edges of $T$ are colored $i(i=1,2,3)$, then

$$
n_{1} \equiv n_{2} \equiv n_{3} \equiv n \quad(\bmod 2)
$$

A minimal 4-coloring of a cubic graph $G$ is an edge-coloring $c: E(G) \rightarrow\{1,2,3,4\}$ such that $\left|c^{-1}(4)\right|$ is minimum. Let $G$ be a cubic graph and let $c: E(G) \rightarrow\{1,2,3,4\}$ be a minimal 4-coloring of $G$. Denote by $E_{i}$ the set of all edges of color 4 which are incident with precisely two edges of color $i$. Since $c$ is minimal, it is easy to see that $\left\{E_{1}, E_{2}, E_{3}\right\}$ is a partition of $c^{-1}(4)$.

The following lemma is a well known consequence of the Parity Lemma (see, e.g., [7]). For the sake of completeness, we include its proof.

Lemma 3.2 Let c be a minimal 4-coloring of a cubic graph $G$. Then $\left|E_{1}\right| \equiv\left|E_{2}\right| \equiv\left|E_{3}\right|$ $(\bmod 2)$.

Proof. Delete from $G$ the edges colored 4. Let $G_{1}$ and $G_{2}$ be two disjoint copies of this graph. Add an edge between every vertex from $G_{1}$ which is of degree two and the corresponding vertex from $G_{2}$. Finally, color each such edge with the free color 1,2, or 3 . We obtain a cubic graph with an edge 3-coloring. There is a cutset of order $2\left(\left|E_{1}\right|+\left|E_{2}\right|+\right.$ $\left|E_{3}\right|$ ) between $G_{1}$ and $G_{2}$. In this cutset, precisely $\left|E_{i+1}\right|+\left|E_{i+2}\right|$ (indices modulo 3) edges are colored $i$ for $i=1,2,3$. By Lemma 3.1, $\left|E_{i+1}\right|+\left|E_{i+2}\right| \equiv 2\left(\left|E_{1}\right|+\left|E_{2}\right|+\left|E_{3}\right|\right) \equiv 0$ $(\bmod 2)$. It follows that $\left|E_{i+1}\right| \equiv\left|E_{i+2}\right| \quad(\bmod 2)$. This completes the proof. 
Proposition 3.3 Let $G$ be a connected simple cubic graph of order $n$, and let $c$ be a minimal 4-coloring of $G$. Then $\left|c^{-1}(4)\right| \leq \frac{1}{5} n$.

Proof. Let $c^{\prime}: E(G) \rightarrow\{1,2,3\}$ be a 3-coloring of $G$, which colors as many edges of $G$ as possible. If $c^{\prime}$ cannot be extended to a 4-edge-coloring of $G$, then we have two incident uncolored edges, say $v u$ and $v w$. Let the third neighbor of $v$ be $z$. We may assume that $c^{\prime}(v z)=3$. Then both colors 1 and 2 are already used at the edges incident with $u$, and the same holds at $w$. Let $P$ be the maximal path which contains the edge $v z$ and whose edges are colored by colors 1 and 3. Note that the other endvertex of this path could be $u$ or $w$. Now, change the color of every edge on $P$ from 1 to 3 , and vice versa. It is not hard to see that we can extend the resulting partial edge coloring of $G$ to $v u$ or $v w$, a contradiction.

So, $c^{\prime}$ can be extended to a 4-edge-coloring $\bar{c}$ of $G$. In particular, $\bar{c}$ is a minimal 4coloring of $G$ and $\left|c^{-1}(\{1,2,3\})\right|=\left|c^{-1}(\{1,2,3\})\right|$. Albertson and Haas [1] proved that such a coloring colors at least $\frac{13}{15}$ of the edges of $G$. Since $c^{\prime}$ colors at least $\frac{13}{15}$ of the edges of $G,\left|c^{-1}(4)\right| \leq \frac{2}{15}|E(G)|=\frac{1}{5} n$.

Theorem 3.4 Let $G$ be a 2-edge-connected graph with $o=o(G)$ odd vertices. Then we can add $\leq\left\lceil\frac{1}{2}\left\lfloor\frac{o}{5}\right\rfloor\right\rceil$ edges such that the new graph admits a nowhere-zero 4-flow.

Proof. Suppose that the claim is false and $G$ is a counterexample with $|E(G)|+|V(G)|$ as small as possible. Let $n=|V(G)|$.

We claim that $G$ is a simple cubic graph. Since $G$ is 2-edge-connected, there are no vertices of degree 1 . It is easy to see that $G$ has no vertices of degree 0 or 2. Otherwise, we obtain a smaller counterexample. Suppose now that $v$ is a vertex in $G$ of degree $\geq 4$. By the Splitting Lemma, we can split this vertex such that the resulting graph is 2-edge-connected. Note that this graph has one or two vertices of degree 2. Let $G^{*}$ be the graph obtained by suppressing the vertices of degree 2. Then, $\left|E\left(G^{*}\right)\right|+\left|V\left(G^{*}\right)\right|<$ $|E(G)|+|V(G)|$ and $o(G)=o\left(G^{*}\right)$. It is easy to see that if we can add at most $\left\lceil\frac{1}{2}\left\lfloor\frac{o}{5}\right\rfloor\right\rceil$ edges to $G^{*}$ in order to obtain a graph which admits a nowhere-zero 4-flow, then we can do it also in $G$. So, $G^{*}$ contradicts the minimality of $G$. This shows that $G$ is a cubic graph. Since $G$ is 2-edge-connected, it has no loops. If it contains a double edge joining vertices $u, v$, we delete one of these edges and obtain a smaller counterexample. This completes the proof of the claim.

Since $G$ is a cubic graph, $n=o$. Let $c$ be a minimal 4-coloring of $G$. By Lemma 3.2, $\left|E_{1}\right| \equiv\left|E_{2}\right| \equiv\left|E_{3}\right| \quad(\bmod 2)$. By Proposition 3.3, $\left|E_{1}\right|+\left|E_{2}\right|+\left|E_{3}\right| \leq\left\lfloor\frac{n}{5}\right\rfloor$.

Suppose first that the sets $E_{i}$ are of even cardinality. Partition each $E_{i}$ into pairs. Consider one of such pairs, $e_{1}=u_{1} v_{1} \in E_{i}$ and $e_{2}=u_{2} v_{2} \in E_{i}$, where the edges incident with $u_{j}$ are colored $i$ and $i+1$ (modulo 3), $j=1,2$. Then, we add the edge $u_{1} u_{2}$ and color it by color $i$. Recolor the edges $e_{1}$ and $e_{2}$ by color $i+1$. We repeat the same procedure for all selected pairs. If we interpret colors $1,2,3$ as the nonzero elements of $\mathbb{Z}_{2} \times \mathbb{Z}_{2}$, we see that we constructed a graph with a nowhere-zero $\mathbb{Z}_{2} \times \mathbb{Z}_{2}$-flow. We have added $\frac{1}{2}\left(\left|E_{1}\right|+\left|E_{2}\right|+\left|E_{3}\right|\right) \leq \frac{1}{2}\left\lfloor\frac{o}{5}\right\rfloor$ edges. 
If $E_{1}, E_{2}, E_{3}$ have odd cardinalities, then we do the same procedure with pairs. At the end, we are left with three edges $e_{i}=u_{i} v_{i} \in E_{i}(i=1,2,3)$. We may assume that edges incident with $u_{i}$ are colored $i$ and $i+1$ (modulo 3). So the colors at $v_{i}$ are $i$ and $i-1$ (modulo 3). Add two edges $v_{1} v_{2}$ and $u_{2} u_{3}$. Now, we color the edges $e_{3}$ and $v_{1} v_{2}$ by color 1 , the edge $e_{2}$ with 2 , and color $e_{1}$ and $u_{2} u_{3}$ by color 3 . As above, we see that we thus constructed a graph with a nowhere-zero $\mathbb{Z}_{2} \times \mathbb{Z}_{2}$-flow. The number of added edges is $\left\lceil\frac{\left|E_{1}\right|+\left|E_{2}\right|+\left|E_{3}\right|}{2}\right\rceil \leq\left\lceil\frac{1}{2}\left\lfloor\frac{O}{5}\right\rfloor\right\rceil$.

\section{5-flows}

Theorem 4.1 Let $G$ be a 2-edge-connected graph with o $=o(G)$ odd vertices. Then we can add $\left\lceil\frac{1}{2}\left\lfloor\frac{o}{7}\right\rfloor\right\rceil$ or fewer edges such that the new graph admits a nowhere-zero 5-flow.

Proof. Suppose that the claim is false and $G$ is a counterexample with $|E(G)|+|V(G)|$ as small as possible. Let $n=|V(G)|$. By the similar arguing as in the proof of Theorem 3.4, we may assume that $G$ is a simple cubic graph.

Now, we will prove that $G$ is of girth $\geq 6$. Let $C=x_{0} x_{1} \cdots x_{r-1} x_{0}$ be a cycle of $G$ with $r$ minimum. Suppose that $r \leq 5$. Let us contract the edges $x_{2 i} x_{2 i+1}$ for $i=0, \ldots,\left\lfloor\frac{r}{2}\right\rfloor-1$. If $r \leq 4$, let $G^{\prime}$ denote the resulting graph. Suppose now that $r=5$. Then we first apply the Splitting Lemma at both new vertices of degree 4 such that $e_{1}=x_{0} x_{4}$ (resp. $e_{1}=x_{3} x_{4}$ ) and such that $e_{0}$ corresponds to the edge of $G-E(C)$ incident with $x_{0}$ (resp $\left.x_{3}\right)$. Denote the resulting graph by $G^{\prime}$. Since $G^{\prime}$ is 2-edge-connected, there are only two possibilities (up to the obvious left-right symmetry) for the structure of $G^{\prime}$ locally at the vertices of $C$. See Figure 1(a) and (b).

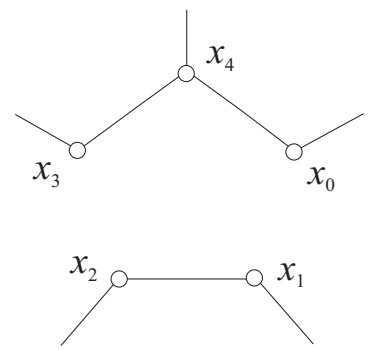

(a)

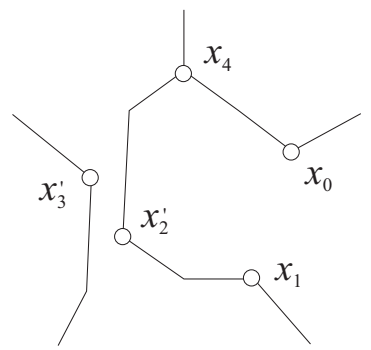

(b)

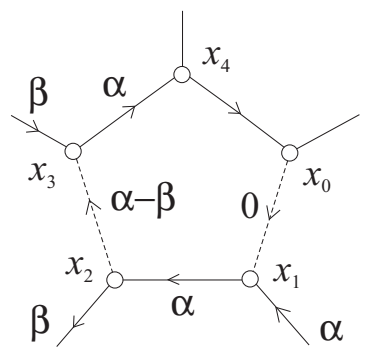

(c)

Figure 1: The two possibilities for $G^{\prime}$ when $r=5$.

By the minimality of $G$, we can prove that $G^{\prime}$ admits a nowhere-zero 5 -flow by adding a set $F$ of at most $\left\lceil\frac{1}{2}\left\lfloor\frac{o-r}{7}\right\rfloor\right\rceil$ edges. Equivalently, $G_{F}^{\prime}$ admits a nowhere-zero $\mathbb{Z}_{5}$-flow $\left(D^{\prime}, \phi^{\prime}\right)$, $\phi^{\prime}: E\left(G^{\prime}\right) \cup F \rightarrow \mathbb{Z}_{5}$. If $r \leq 4$, then $\phi^{\prime}$ determines a $\mathbb{Z}_{5}$-flow $\left(D, \phi_{1}\right)$ of $G_{F}$ which agrees with $\left(D^{\prime}, \phi^{\prime}\right)$ on $E\left(G^{\prime}\right) \cup F$. Note that $\phi_{1}$ is nonzero on $(E(G) \cup F) \backslash E(C)$. If $r=5$, then we claim that $\phi^{\prime}$ determines a $\mathbb{Z}_{5}$-flow $\left(D, \phi_{1}\right)$ of $G_{F}$ which agrees with $\left(D^{\prime}, \phi^{\prime}\right)$ on 
$\left(E(G) \cap E\left(G^{\prime}\right)\right) \cup F$, which is nonzero on $(E(G) \cup F) \backslash E(C)$, and such that $\phi_{1}$ takes at most four distinct values on $E(C)$, where all edges of $C$ are assumed to be oriented "clockwise". In the first case of Figure 1, the claim is obvious. We just set $\phi_{1}(e)=\phi^{\prime}(e)$ for $e \in E\left(G^{\prime}\right) \cup F$ and set $\phi_{1}\left(x_{0} x_{1}\right)=\phi_{1}\left(x_{2} x_{3}\right)=0$. In the second case of Figure 1, we consider the edges $x_{1} x_{2}^{\prime}, x_{2}^{\prime} x_{4}$, and the two edges incident with $x_{3}^{\prime}$ as being the edges $x_{1} x_{2}$, $x_{3} x_{4}$, and edges incident with $x_{2}$ and $x_{3}$, respectively, as indicated in Figure 1(c). The flow condition may be violated at $x_{2}$ and $x_{3}$ but there is a unique value for $\phi_{1}\left(x_{2} x_{3}\right)$ such that we get a flow. (Also, we set $\phi_{1}\left(x_{1} x_{5}\right)=0$.) All vertices of $C$ except $x_{4}$ give rise to vertices of degree 2 in $G^{\prime}$. Therefore, no edges of $F$ are incident with them. This implies that the $\phi_{1}$-flow on the edges $x_{1} x_{2}$ and $x_{3} x_{4}$ is the same as the $\phi^{\prime}$-flow through the vertex $x_{2}^{\prime}$ of $G^{\prime}$. Consequently, $\phi_{1}$ takes at most four distinct values on $E(C)$.

Returning to the general case $r \leq 5$, let $i \in \mathbb{Z}_{5}$ be a value which does not occur as a $\phi_{1}$-value on $E(C)$. Recall that the orientation $D$ orients $C$ clockwise. So, there is a $\mathbb{Z}_{5}$-flow $\left(D, \phi_{2}\right)$ of $G_{F}$ which is 0 on $(E(G) \backslash E(C)) \cup F$ and such that $\phi_{2}(e)=i$ for edges on $C$. Now, $\left(D, \phi_{1}-\phi_{2}\right)$ is a nowhere-zero $\mathbb{Z}_{5}$-flow of $G_{F}$. This contradiction shows that $r \geq 6$.

Since $G$ is a 2-edge-connected cubic graph, it has a 2-factor $Q$ by the Petersen theorem. Since every cycle in $Q$ is of length $\geq 6$, we can color at least $\frac{6}{7}$ of the edges of $Q$ using colors 1 and 2 . Color every edge of the 1-factor $E(G)-E(Q)$ by color 3 . Thus, we have a partial 3-edge-coloring of $G$, which colors at least $\frac{19}{21}$ of the edges of $G$. So, the number of uncolored edges is $\leq\left\lfloor\frac{2}{21}|E(G)|\right\rfloor=\left\lfloor\frac{o}{7}\right\rfloor$.

In a similar way as in Theorem 3.4, we can add at most $\left\lceil\frac{1}{2}\left\lfloor\frac{o}{7}\right\rfloor\right\rceil$ edges to $G$ in order to a obtain a graph which admits a nowhere-zero 5-flow. (In fact, we even get a nowhere-zero 4-flow in this case.)

\section{Concluding remarks}

We will conclude the paper with the following remarks. First, in all results of the paper, we are restricted to 2-edge-connected graphs. It is not hard to construct graphs with cutedges for which bounds from the theorems are not valid.

Another obvious question is: "How good is the bound of Theorem 4.1." The 5-FlowConjecture of Tutte [9] namely asserts that $\phi_{5}^{+}=0$ for every 2-edge-connected graph. The following proposition answers this question.

Proposition 5.1 Let $k \in\{3,4,5\}$. For every integer s there is a 2-edge-connected graph $G$ with $o(G) \geq$ s such that

(a) If $k=3$, then $\phi_{k}^{+}(G) \geq \frac{1}{8} o(G)$.

(b) If $k=4$, then $\phi_{k}^{+}(G) \geq \frac{1}{20} o(G)$.

(c) If $k=5$, and the 5-Flow-Conjecture is false, then there is a constant $c>0$ such that $\phi_{k}^{+}(G) \geq c \cdot o(G)$. 
Proof. Let $G$ be a 2-edge-connected graph without a nowhere-zero $k$-flow. Let $e=u v$ be an edge of $G_{0}$. Take $s$ copies of $G_{0}-e$ and form the graph $G$ by joining the copy $v_{i}$ of $v$ in the $i^{\text {th }}$ copy of $G_{0}-e$ with the copy $u_{i+1}$ of $u$ in the $(i+1)^{\text {st }}$ copy of $G_{0}-e, i=1,2, \ldots, s$ (indices modulo $s$ ). Then $G$ is 2-edge-connected and $o(G) \geq s$. If $\phi_{k}^{+}(G)<\frac{s}{2}$, then there is an edge set $F$ such that $G_{F}$ has a nowhere-zero $k$-flow and there is a copy of $G_{0}-e$ such that no edge of $F$ is incident with its vertices. Then it is easy to see that the flow of $G_{F}$ gives rise to a nowhere-zero $k$-flow of $G_{0}$, a contradiction. This shows that

$$
\phi_{k}^{+}(G) \geq \frac{s}{2} \geq \frac{1}{2\left|V\left(G_{0}\right)\right|} o(G) .
$$

Finally, let $G_{0}=K_{4}$ if $k=3$, let $G_{0}$ be the Petersen graph if $k=4$, and let $G_{0}$ be a hypothetical counterexample to the Tutte 5-Flow-Conjecture if $k=5$. Then (1) implies the proposition.

Let $k \in\{2,3,4,5\}$. One can ask how hard it is to calculate $\phi_{k}^{+}(G)$ and $\phi_{k}^{-}(G)$ for a given graph $G$. As we already said, $\phi_{2}^{+}(G)=\frac{o(G)}{2}$. Calculating $\phi_{2}^{-}(G)$ is equivalent to finding a Chinese postman tour in $G$ (see Lemma 8.1.4 in [10]). Edmonds and Johnson [3] proved that the Chinese postman problem is solvable by a polynomial time algorithm. The decision problem whether $\phi_{4}^{+}(G)=0$ is an NP-complete problem. This follows by the fact that it is an NP-complete problem to decide whether a (cubic) graph is 3-edge-colorable.

The decision whether $\phi_{5}^{+}(G)=0$ or not is either trivial (if the 5-Flow-Conjecture holds) or NP-complete, as proved by Kochol [5]. Similar conclusion holds for 3-flows, depending on the Tutte 3-Flow-Conjecture (cf. Kochol [5]).

\section{References}

[1] M. O. Albertson and R. Haas, Parsimonious edge colorings, Discrete Math. 148 (1996) 1-7.

[2] D. Blanuša, Problem četeriju boja (The problem of four colors), Math.-Fiz. Astr. Ser. II (1) (1946) 31-42.

[3] J. Edmonds and E. L. Johnson, Matchings, Euler tours and the Chinese postman, Math. Progr. 5 (1973) 88-124.

[4] H. Fleischner, Eine gemeinsame Basis für die Theorie der Eulerschen Graphen und der Satz von Petersen, Monatsh. Math. 81 (1976) 267-278.

[5] M. Kochol, Hypothetical complexity of the nowhere-zero 5-flow problem, J. Graph Theory 28 (1998) 1-11.

[6] P. D. Seymour, Nowhere-zero 6-flows, J. Combin. Theory B 30 (1981) 130-135.

[7] E. Steffen, Classifications and characterizations of snarks, Discrete Math. 188 (1998) 183-203. 
[8] W. T. Tutte, On the imbedding of linear graphs in surfaces, Proc. London Math. Soc. 51 (1954) 474-483.

[9] W. T. Tutte, A contribution to the theory of chromatic polynomials, J. Canad. Math. Soc. 6 (1954) 80-91.

[10] C.-Q. Zhang, Integer flows and cycle covers of graphs, Marcel Dekker Inc., New York, 1997. 